\title{
Mining IPBES assessments
}

\author{
Scholars contributing to the Intergovernmental Science-Policy Platform on Biodiversity and Ecosystem Services \\ (IPBES) have a lot of in-depth insights to share. Here's what we recommend to those who are willing to do it.
}

$\mathrm{n}$ the first week of May 2019,
representatives from the governments
of over 130 countries gathered at the UNESCO headquarters in Paris to agree, one by one, on all the statements of the summary of the IPBES Global Assessment, published on 6 May. This is IPBES' latest assessment on the extent and drivers of biodiversity loss, and the most comprehensive to date. The report also tells policymakers and the wider public of possible pathways to revert this loss. Over more than four years, hundreds of academics have reviewed and synthesized the available evidence. And such evidence is vast. Nature Sustainability is open to considering articles that reflect the deep understandings acquired by reviewing such material and that would add value to the assessments, and encourage authors to get in touch.

IPBES is an intergovernmental platform created to bridge science and policy on biodiversity and ecosystems, supporting the implementation of the Convention on Biological Diversity (the main multilateral agreement on the topic). IPBES is joined by hundreds of academics and civil-society representatives across the globe. This intergovernmental and scientific initiative around sustainability is said to be the most inclusive to date, in terms of representatives' geographical and gender diversity (see the Review by Díaz-Reviriego et al. in this issue).

The latest assessment is no usual document. While the dire prospects of biodiversity and the biosphere are not news to most of our readers, an assessment commissioned by an intergovernmental body, supported by scientists, and adopted by government officials, can influence the international community. Governments can now use this evidence to put forward far more ambitious plans to save life on the planet.

Essentially, these assessments are knowledge reviews of unprecedented coverage, including peer-reviewed and grey literature, and traditional knowledge. The evidence touches most sustainability matters including land-use and climate change, water scarcity or indigenous and local knowledge about nature. The breadth and depth of understanding attained by this reviewing effort isn't fully reflected in the official IPBES documents, and we believe that there is room to develop more scholarly articles that complement the assessments and foster further discussion about the

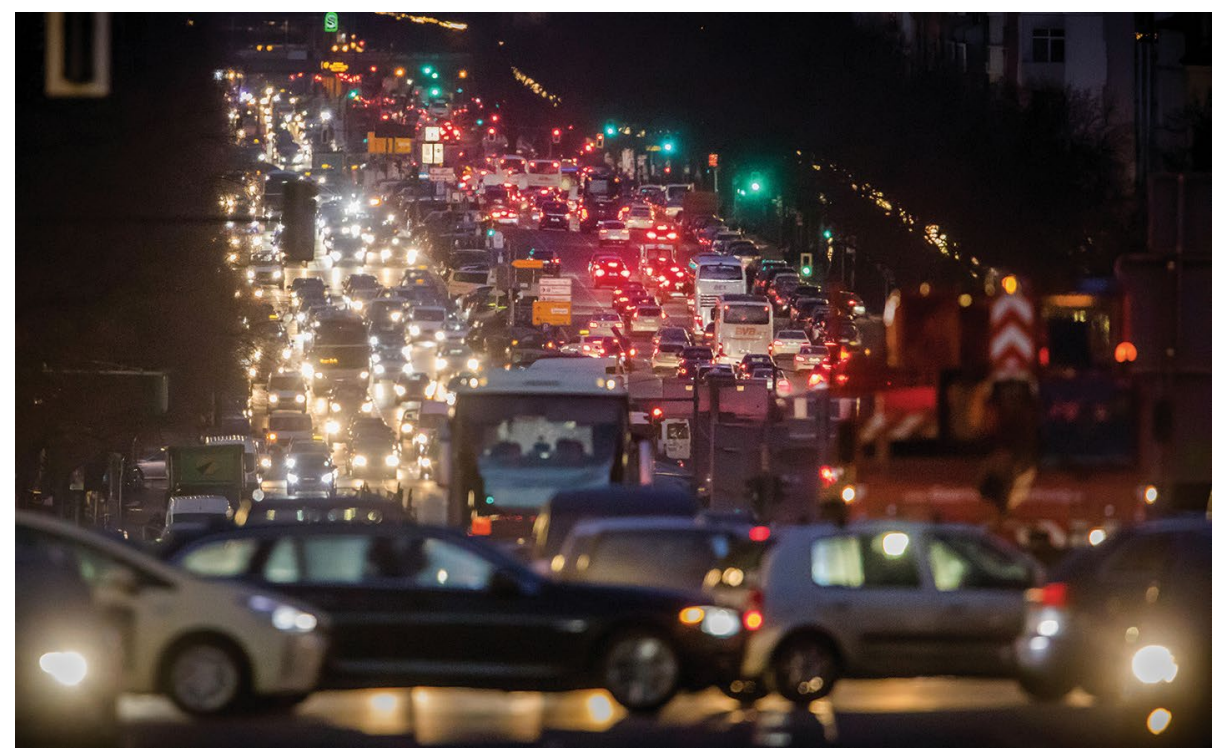

Credit: dpa picture alliance / Alamy Stock Photo

biodiversity crisis across domains. Here we give some general guidelines that we hope will assist those authors willing to mine the IPBES reports they have contributed to, and consider publishing important outputs in Nature Sustainability.

We expect articles to be standalone and not akin to sections of the reports. Such articles will discuss relevant topics through a much more focused lens than the overarching framing of the reports or of parts of them. As a result, they will move beyond citing the reports to carefully select specific references in support of the arguments presented. The article scope will be more contained than the extensive breadth of the assessments. More focused articles will encourage deeper discussions than a plain literature synthesis. Ultimately they will offer new insights that readers would not be able to grasp from the official reports, including through original display items.

How can they be formatted? Depending on the goal of the article and whether new analysis is presented, authors can consider either a Review or an original research article, and the basic descriptions can be found here. Of course, we can provide feedback on proposals and we encourage authors to submit ideas in the form of a summary as a pre-submission enquiry, through our platform. In addition, there could be opportunities to write opinion pieces. These would work when authors focus on an ongoing issue and propose a specific, concrete and implementable pathway to address it, from a personal viewpoint.

Authors may want to provide policy recommendations of interest to many and not exclusively in the IPBES domain. Regardless of article type, any policy recommendations should discuss a specific implementation path. This is vital to effectively contribute to broader debates. For example, multilevel and participatory governance is often recommended. But what should practitioners do to make this happen? Generic statements about potentially useful approaches will not compel most readers. Policy proposals should stimulate further scholarly thought, and indicate possible and realistic ways to translate into actions. They should do so in light of the complexity of policymaking and the many forces that are driving the loss of the richness and diversity of life on Earth.

Published online: 11 June 2019

https://doi.org/10.1038/s41893-019-0321-3 